\title{
A Low-Cost, Low-Delay Location Update/Paging Scheme in Hierarchical Cellular Networks
}

\author{
Xiaoxin $\mathrm{Wu}$ \\ Dept. of Computer Sciences \\ Purdue University \\ West Lafayette, IN47906 \\ wu@cs.purdue.edu
}

\author{
Biswanath Mukherjee \\ Dept. of Computer Science \\ University of California \\ Davis, CA 95616 \\ mukherje@cs.ucdavis.edu
}

\author{
Bharat Bhargava \\ Dept. of Computer Sciences \\ Purdue University \\ West Lafayette, IN47906 \\ bb@cs.purdue.edu
}

\begin{abstract}
A low-cost, two-step location update/paging scheme in a macrocell/microcell network is proposed and investigated. To reduce operating cost, the location update is operated only in the macrocell tier. A callee will be paged in the macrocell tier first. If the paging delay in the macrocell tier is too high due to large queuing delay, the callee will then be paged in the microcell tier. Original searching method is used in the microcell tier paging. The operation for the scheme is simple, since the macrocell/microcell cellular network has the advantage that a mobile user in such a cellular network can receive a signal from both a macrocell and a microcell. The analytical results show that, along with the low location update/paging cost, the two-step paging scheme also achieves low paging delay.

Categories and Subject Descriptors: C.2.1 [ComputerCommunication Networks]: Network Architecture and Design
\end{abstract}

General Terms: Design.

Keywords: macrocell/microcell, paging, location update, hierarchical cellular network, quality of service

\section{INTRODUCTION}

Location update/paging constitutes an important mobility problem in a cellular network, especially when cells are becoming smaller and the number of mobile users is growing rapidly. In the existing mobile cellular networks, such as a GSM network, a mobile user initially registers with a location area (LA) managed by its home location register (HLR). An LA is combined of a number of cells. When the mobile user moves out of its the LA, a location update is needed. If it moves out of the area managed by its HLR, it has to register in a visiting location register (VLR). This

Permission to make digital or hard copies of all or part of this work for personal or classroom use is granted without fee provided that copies are not made or distributed for profit or commercial advantage and that copies bear this notice and the full citation on the first page. To copy otherwise, to republish, to post on servers or to redistribute to lists, requires prior specific permission and/or a fee.

MobiDE'03, September 19, 2003, San Diego, California, USA.

Copyright 2003 ACM 1-58113-767-2/03/0009 ...\$5.00.
VLR will inform the mobile user's HLR about the mobile user's registration.

A mobile user is paged in the following sequence: the paging message is sent to the mobile user's HLR. If the mobile user is out of the area managed by HLR, the HLR will forward this paging message to its VLR. The paging message is broadcast on the paging channels of all the cells in the current LA of the mobile user, so that the paged user can be found.

The cost of the location update/paging process is mainly the radio bandwidth consumption in the wireless channels and the signaling exchange in the core network. A major concern for efficient mobility management in the cellular network is keeping the location update/paging cost low while maintaining a low paging delay.

This paper investigates the location update/paging schemes with low cost and low paging delays in the macrocell/microcell cellular network, the hierarchical cellular architecture introduced to improve the system capacity [1]. In such a network, since a macrocell covers a much larger area than a microcell, making up LAs by macrocells results in much fewer LAs in the network. The location-update cost for each mobile user can be reduced, while the paging cost does not increase if considering that the radio bandwidth in the macrocell tier has the same value as that in the microcell tier (In a real network, the bandwidth a in macrocell is normally more precious than that in a microcell). However, since both the macrocell users and the microcell users in an LA are paged only through the paging channels of the macrocells, the overall paging load in the macrocell tier may become very high. This is primarily because the number of microcell users is much larger than the number of macrocell users. In this case, the paging messages may encounter long queuing delay in the macrocells' paging channels and the delay requirement may not be satisfied. On the other hand, if we reduce the size of LAs by making up LAs by microcells, the paging load is divided into smaller areas with less load in each area. The paging delay decreases, yet the location-update cost increases because mobile users may have to experience more location updates.

To solve this dilemma, we propose a new location update/paging scheme, called two-step paging scheme, for macrocell/microcell networks. The low paging delay location update/paging strategies for the single-tier cellular network can 
be applied to the macrocell/microcell network with appropriate modifications. In addition, the hierarchical structure of such a network can bring more flexibility in location update/paging management. The proposed scheme uses the unique feature of the macrocell/microcell cellular network, namely that a mobile user can receive signals from both a microcell and the overlaid macrocell. A macro/micro hierarchical LA is used, and location update is operated only in the macrocell tier. The LA structure is similar to the multilayer LAs in [2], but with a simpler operation. For paging, a mobile user is paged in the macrocell tier first. If the queue of paging messages in the macrocell tier is too long, the mobile user will then be paged in the microcell tier. Different paging strategies are used in the macrocell tier and the microcell tier. This scheme can be easily implemented in a cellular network with channels divided into time slots. The paging channels of a microcell and its overlaid macrocell may use different time slots. Mobile users adjust their receivers periodically to listen to the paging channels from different tiers at different times, so they can receive the paging messages from both the macrocell tier and the microcell tier. The operation of such a scheme is described in Section 2.

Previous research has a focus on minimizing the cost of location update/paging while meeting the paging delay requirement in the single-tiered network. The proposed methods can also be used in the hierarchical cellular network. These work can be found in $[3,4,5]$. Location update and paging in hierarchical cellular networks are studied in [6], [7].

Section 2 describes the two-step paging scheme and two other basic paging schemes for comparison. In section 3, analytical models are developed for the paging delay and the location update/paging cost. Section 4 shows illustrative results. Section 5 concludes our work.

\section{TWO-STEP PAGING SCHEME}

In this section, the two-step paging scheme (Scheme C) is described. Two basic location update/paging schemes in the macrocell/microcell network (Scheme A and Scheme B) are used to compare with our proposed scheme. It is assumed that within one LA, in each paging slot, only one paging message is broadcast. Paging messages will stay in a first-infirst-out (FIFO) queue to wait for the next paging slot if they cannot be processed immediately. It is also assumed that, with the location update, the LA with which a mobile user registers is known exactly, and the paged user can be found after the first paging. The three location update/paging schemes in the macrocell/microcell network which our work compares are as follows:

- Scheme A: Location update/paging is only operated in the microcell tier. In this scheme, each LA consists of a number of microcells, and we call the LA a micro-LA. Mobile users judge whether their microLAs change by listening to the microcells' broadcast channels. When a mobile user's micro-LA changes, a location update is needed. A mobile user is paged simultaneously in all the microcells within its registered micro-LA (a home LA or a visiting LA).

- Scheme B: Location update/paging is only operated in the macrocell tier. In this scheme, each LA consists of a number of macrocells, and we call the LA a macroLA. The paging and location-update process is similar to that in Scheme A, but operated in macro-LAs.

- Scheme C (two-step paging scheme): Location update is operated in the macrocell tier, and mobile users are paged in both the macrocell tier and the microcell tier. In this scheme, the macrocell tier is divided into macro-LAs and the microcell tier is divided into microLAs. Each macro-LA overlaps a number of micro-LAs, as shown in Figure 1. Mobile users register with macroLAs and location update is only operated in the macrocell tier, which means location update is needed only when a mobile user's macro-LA changes. A mobile user is initially paged in its macro-LA, by which the callee can be found after the paging message is broadcast once. If the paging queue is too long, this mobile user will then be paged in the underlying microLAs. Since the exact micro-LA of the paged mobile user is not known, the original search method is used in the microcell tier, i.e., the mobile user is paged in the micro-LAs one by one until it is found. Note that paging strategies such as that in [4] can be used in the microcell tier to improve the system performance. The original search method is used here for simplicity.

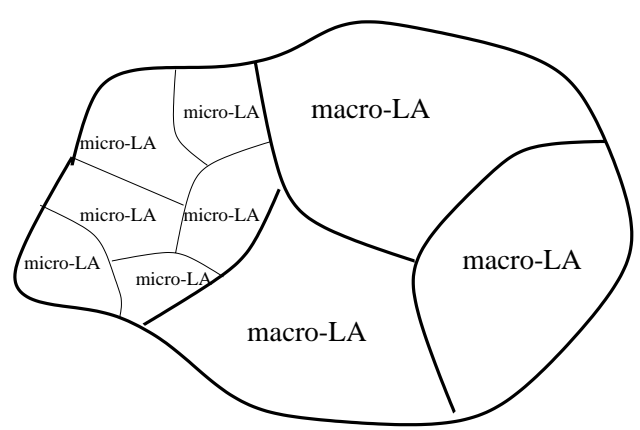

Figure 1: Macro-LAs and micro-LAs in a macrocell/microcell network.

In the two-step paging scheme, an idle mobile user has to listen to the paging channels of both tiers. In a time-slotted tiered network, if both tiers occupy the same radio frequency, the mobile user may just listen to the paging channels in different tiers at different time slots. If different frequencies are used in macrocell tier and microcell tier, as is normally the case in a TDMA tiered network, the time interval between a macro-LA paging slot and the paging slots of its underlying micro-LAs should be long enough for a mobile user to adjust its receiver to different frequencies. In the tiered network, since the frequency adjustment is needed for mobile users to receive the broadcast signals from all the tiers, the two-step paging scheme does not bring much additional operating overhead.

\section{ANALYSIS OF DELAY AND COST}

We first define the key network parameters that will be used in the analysis. We then build the mathematical model for paging delay. Finally, we analyze the average cost for a 
single location update/paging process in the macrocell/microcell cellular network.

\subsection{Network Architecture, Parameters, and As- sumptions}

- In a macrocell/microcell cellular network, each macroLA is made up by $N_{a}$ macrocells and each micro-LA is made up by $N_{i}$ microcells. Each macrocell is overlaid with $N_{m c}$ microcells. Thus, a macro-LA overlaps with $N_{m l}$ micro-LAs, where $N_{m l}=N_{a} \times N_{m l} / N_{i}$.

- A homogeneous environment and the paging messages coming to a micro-LA follow a Poisson distribution with an arrival rate of $\lambda_{m i}$ messages/sec is assumed. The overall paging messages coming to a macro-LA, $\lambda_{m a}$, also follow a Poisson distribution with the arrival rate of $N_{m l} \lambda_{m i}$ messages/sec.

- We assume the time that a mobile user will stay within the same micro-LA is negative exponentially distributed with mean $1 / \mu_{m i}$ seconds. From [8], $\mu_{m i}$ can be calculated by:

$$
\mu_{m i}=\frac{\pi S}{E[v] L}
$$

where $S, L$ are the area and the perimeter of the micro-LA and $E[v]$ is the average speed of this mobile user. Assuming all LAs are hexagonal (or approximately circle-shaped), since each macro-LA covers $N_{m l}$ micro-LAs, by Eqn. (1), the time that a mobile user stays in a macro-LA then is also negative exponentially distributed with mean $1 / \mu_{m a}$ seconds, and $\mu_{m a}=\mu_{m i} / \sqrt{N_{m l}}$.

- For a mobile user, we assume that the inter-arrival time between any two consecutive calls to it is Poisson distributed with a mean inter-arrival time of $1 / \mu_{c}$ seconds. To simplify the analysis, we assume that this time is much larger than the average call-holding time and there is no location update during the mobile user's talking process.

\subsection{Average Delay}

Let $T$ be the time between two paging slots (a paging slot is normally much smaller than $T$ ). Since, we assumed earlier, that only one paging message is processed in a paging slot $^{1}$ and the new paging messages will stay in the queue waiting for paging slots, the paging process can be described as a discrete Markov chain with the embedded time of $T$. Assuming the Poisson arrival with a arrival rate of $\lambda$ for paging messages, the paging process is a $M / D / 1$ process, with a fixed service time $T$. Define $p(i)$ the probability that $i$ paging messages are queued, then $p(i)$ can be calculated following the routing queuing theory.

For this queuing system with a finite queue length of $n$, define $\bar{k}$ to be the average number of users in the system, then:

$$
\bar{k}=\sum_{i=0}^{i=n} i p(i) .
$$

${ }^{1}$ In the real GSM system, a paging block can normally process more than 1 paging messages. We assume one paging message per slot for analytical tractability.
Using Little's law, the average delay $D$ is:

$$
D=\frac{\bar{k}}{\lambda(1-p(n))} .
$$

For the paging Schemes A and B, the average delay can be calculated using Eqn. (3) directly when $n$ is large enough.

In Scheme C, a mobile user is paged in its macro-LA first. To satisfy the delay requirement, we assign to the macro-LA a finite paging queue with length no longer than $Q$. From Eqn. (3), we can find $D_{m a}$, the average paging delay in the macrocell tier. The overflow from the macrocell tier to the microcell tier can be approximated to follow a Poisson distribution. Since the paging message arrival rate to the macro$\mathrm{LA}$ is $\lambda_{m a}$, when the queue length in the macro-LA is $Q$, define $\lambda_{o v}$ to be the overflow rate to the microcell tier; then:

$$
\lambda_{o v}=\lambda_{m a} p(Q) .
$$

Assume that the paged users are uniformly distributed in the network. Knowing a paged user's macro-LA, and if this user is paged in micro-LAs, the probability that the paged user can be found in each micro-LA is $1 / N_{m l}$, where $N_{m l}$ is the number of micro-LAs overlaid by the macro-LA. For each paging message broadcast in the microcell tier, we define $\bar{N}$ to be the average number of times it is broadcast in the micro-LAs until the paged user is found; then:

$$
\bar{N}=\sum_{i=1}^{N_{m l}} i \frac{1}{N_{m l}}=\frac{N_{m l}+1}{2} .
$$

Among all the micro-LAs overlaid by the same macroLA, if the network starts searching for a paged user in a randomly-picked micro-LA and with a random searching sequence among the micro-LAs, for analysis tractability, we approximate the paging flow to each micro-LA by a Poisson distribution with arrival rate of $\lambda_{a-i}$, and:

$$
\lambda_{a-i}=\frac{\bar{N} \lambda_{o v}}{N_{m l}} .
$$

Define the average waiting time in each micro-LA to be $D_{m i}$; then $D_{m i}$ can be calculated through Eqn. (3). Since a paging message will be broadcast for the average of $\bar{N}$ times, the overall average processing delay in the microcell tier is $\bar{N} D_{m i}$. Define $D_{S C}$ to be the average processing delay for the two-step paging scheme; then:

$$
D_{S C}=D_{m a}\left(1-p_{o v}\right)+\bar{N} D_{m i} p_{o v} .
$$

In Scheme $\mathrm{C}$, the paging messages can only encounter large delay in the microcell tier. Define $f_{p}(k)$ to be the discrete probability-distribution function for the probability that when a new paging message comes to a micro-LA, there are already $k$ queued paging messages in this microLA. Since a new paging message will experience an average of $\bar{N}$ broadcasts, the paging process for this paging message in the micro-LAs can be approximated as follows: this paging message comes to an equivalent queue with $n$ messages in the queue, of which the discrete probability density function $P(k)$ for this equivalent queue (defined as the probability that there are $n$ paging messages in the equivalent queue) can be calculated by:

$$
P(k)=p_{1}(k) \star p_{2}(k) \star p_{3}(k) \cdots \star p_{N}(k),
$$


where $\star$ is the symbol for convolution and $p_{1}(k)=p_{2}(k)=$ $\cdots=p_{N}(k)=p(k)$.

\subsection{Cost}

We define $w_{p a}$ and $w_{p i}$ to be the paging cost when one paging message is broadcast in a macrocell and microcell respectively, and $w_{u}$ to be the cost for one location update of one mobile user. The location update/paging cost we try to find is the paging cost for a mobile user, and the location update cost on this mobile user since last time it is called (i.e., the cost of location update for this mobile user between any two consecutive calls to it).

When location update is operated in the microcell tier (e.g., in Scheme A), we define $t_{1}$ to be the time that a mobile user will stay in a micro-LA and $t_{2}$ to be the time interval between two consecutive calls to this mobile user. Both $t_{1}$ and $t_{2}$ are negative exponentially distributed with mean $1 / \mu_{m i}$ and $1 / \mu_{c}$, respectively. A location update is needed when $t_{2}>t_{1}$. Defining $p_{u}$ to be the probability that there is at least one location update between any two consecutive calls to this mobile user, and defining $f_{t_{1}}\left(t_{1}\right), f_{t_{2}}\left(t_{2}\right)$ to be the $p d f$ functions for $t_{1}, t_{2}$, we have:

$$
\begin{aligned}
f_{t_{1}}\left(t_{1}\right) & =\mu_{m i} e^{-\mu_{m i} t_{1}} \\
f_{t_{2}}\left(t_{2}\right) & =\mu_{c} e^{-\mu_{c} t_{2}}
\end{aligned}
$$

In Scheme $\mathrm{A}, p_{u}$ can be calculated by:

$$
\begin{aligned}
p_{u}=p\left[t_{2}>t_{1}\right] & =\int_{0}^{\infty} \int_{t_{1}}^{\infty} f_{t_{2}}\left(t_{2}\right) f_{t_{1}}\left(t_{1}\right) d t_{2} d t_{1} \\
& =\int_{0}^{\infty} \int_{t_{1}}^{\infty} \mu_{c} e^{-\mu_{c} t_{2}} \mu_{m i} e^{-\mu_{m i} t_{1}} d t_{2} d t_{1} \\
& =\frac{\mu_{m i}}{\mu_{m i}+\mu_{c}}
\end{aligned}
$$

Note that the exponential distribution has the memoryless feature. In Scheme A, since a mobile user is called the last time, the probability that this mobile user's location has to be updated by $n$ times before it is called another time is $p_{u}^{n}$. Between any two consecutive calls, define the average number of location updates this mobile user experiences as $\bar{N}_{S A}$, then:

$$
\bar{N}_{S A}=\sum_{i=1}^{\infty} i p_{u}^{i}=\frac{p_{u}}{\left(1-p_{u}\right)^{2}} .
$$

Similarly, by using $\mu_{m a}$ instead of $\mu_{m i}$ in Eqn. (9), we can find the average number of location updates between any two consecutive calls in Schemes B and C by Eqn. (10). We define the numbers as $\bar{N}_{S B}$ and $\bar{N}_{S C}$, and $\bar{N}_{S B}=\bar{N}_{S C}$.

For Schemes A and B, a mobile user will be paged once since the mobile user will be found after the first paging. For Scheme C, in the macrocell tier, a mobile user will be paged once and in the microcell tier, a mobile user is paged an average of $\left(N_{m l}+1\right) / 2$ (from Eqn. (5)) times. Since $p(Q)$ is the probability that a mobile user has to be paged in the micro-LAs in Scheme $\mathrm{C}$ since all $Q$ queue positions in the overlaid macro-LA are taken, define $C_{S A}, C_{S B}$, and $C_{S C}$ to be the average location update/paging cost for a mobile user in Scheme A, B, and C; then:

$$
\begin{aligned}
C_{S A}= & N_{i} w_{p i}+\bar{N}_{S A} w_{u} \\
C_{S B}= & N_{a} w_{p a}+\bar{N}_{S B} w_{u}, \\
C_{S C}= & w_{p a}(1-p(Q))+\frac{N_{m l}+1}{2} w_{p i} p(Q) \\
& +\bar{N}_{S C} w_{u} .
\end{aligned}
$$

\section{ILLUSTRATIVE NUMERICAL EXAMPLES}

In this section, we show some illustrative results on the delay and cost. In our examples here, each macrocell is overlaid 7 microcells. Each macro-LA contains 7 macrocells and each micro-LA contains 7 microcells. Thus, in Scheme C, each macro-LA covers 7 micro-LAs. This architecture is for simplicity, and the optimum architecture can be found by simulation. We only consider signaling cost and use the cost in [9] for paging and location update cost estimation. For paging, the cost is 463 bit for each cell and for location update, the cost is 1231 bit for a single location update. We assume the paging cost in a microcell is the same as that in a macrocell. The interval time between any two paging slots is normalized as 1, i.e., $T=1$.

Figure 2 shows the delay in Scheme $\mathrm{C}$ when the macroLA's paging queue has different lengths (different $Q \mathrm{~s}$ ). When load is high, schemes with longer queue have larger delay because, in such schemes, more calls are served in the macrocell tier, where the average paging delay is high at high load. The average delay decreases when queue length decreases at high load. However, when queue length is very short, e.g., $Q=2$, the delay will become larger at low load because more paging messages have to overflow to the microcell tier, where a single paging message may be broadcast several times.

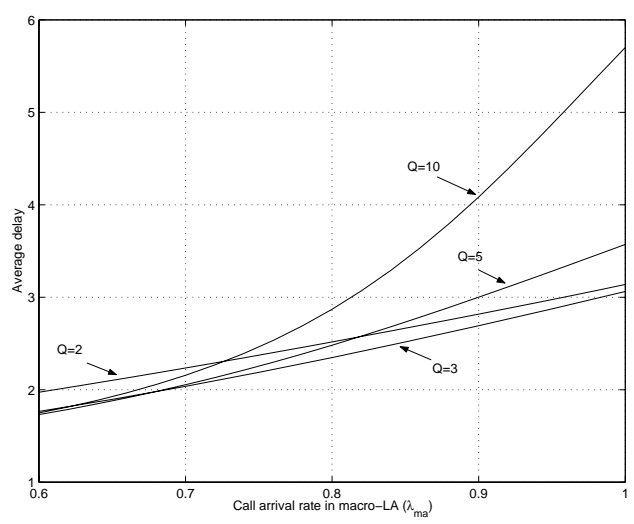

Figure 2: Average delay in Scheme $\mathrm{C}$ with different queue lengths.

In Figure 3, we show the cost for Scheme $\mathrm{C}$ with different queue length in the macro-LA. The $\mathrm{x}$ axis $\left(\mu_{m a} / \mu_{c}\right)$ shows how often a mobile user is called within a macro-LA (How often a mobile user is called within a micro-LA can be similarly described as $\mu_{m i} / \mu_{c}$.). When $\mu_{m a} / \mu_{c}$ increases, a mobile user is called less often within the same macro-LA and there is a larger probability that a location update is needed between two consecutive calls to this mobile user. Taking consideration the heavy paging load in the macro-LA, it is assigned a paging load $\lambda_{m a}$ of 0.95 . It is shown that with 
a smaller queue length, the overall location update/paging cost is higher because more paging messages will overflow to the microcell tier, where the paging cost is higher.

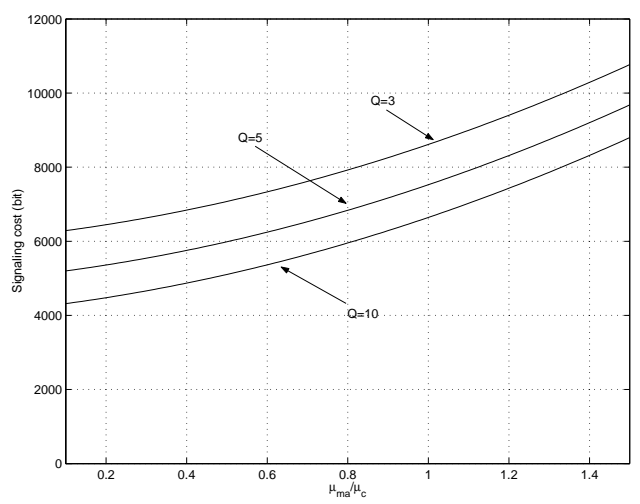

Figure 3: Location update/paging cost for Scheme C with different $\mu_{m a} / \mu_{c}$.

We assign the paging queue length in the macro-LA in Scheme $\mathrm{C}$ as 5 , so that the scheme has relatively good performance in both delay and cost. The average paging delays for Schemes A, B, and C are shown in Figure 4. The average delay in Scheme B is much larger than that in Scheme A since the paging load in each micro-LA is much smaller than that in each macro-LA. The average paging delay in Scheme C is marginally worse than that in Scheme A, but better than that in Scheme B especially when paging load in a macro-LA is high.

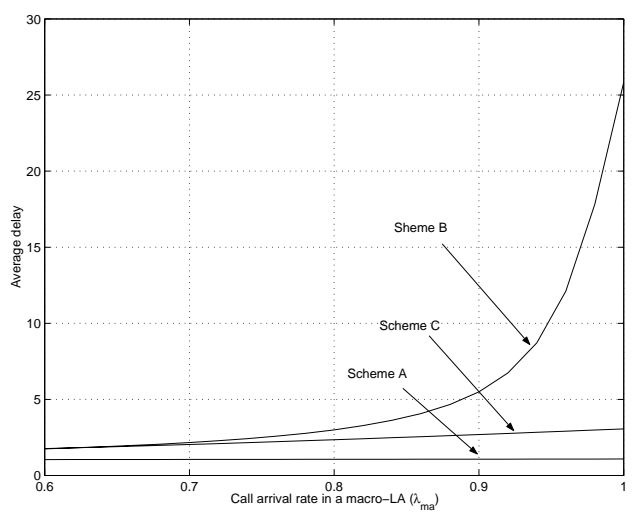

Figure 4: Paging delay in Schemes A, B, and C.

We show in Figure 5 the cost comparison among different schemes. It is observed that, although Scheme A has the smallest delay in the previous result, it has much larger cost than Schemes B and C. The cost of Scheme C is only marginally larger than that of Scheme B. It can also be observed from this figure that the cost increases when $\mu_{m a} / \mu_{c}$ increases since a larger $\mu_{m a} / \mu_{c}$ causes more location updates between any two consecutive calls.

\section{CONCLUSION}

In this work, we examined a new location update/paging scheme in a macrocell/microcell cellular network, called a

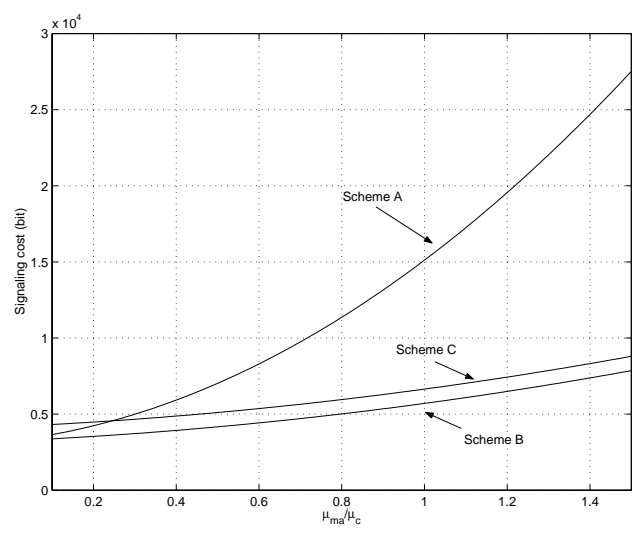

Figure 5: Location update/paging cost in Schemes $\mathrm{A}, \mathrm{B}$, and $\mathrm{C}$.

two-step paging scheme. A location update is only operated in the macrocell tier to reduce the cost. A mobile user is paged in the macrocell tier first. If the waiting queue is too long in the macrocell tier, the mobile user will then be searched in the underlying microcells to reduce the paging delay. With the special feature that, in a hierarchical cellular network, a mobile user can reach the base stations from both the tiers, the two-step paging scheme demonstrates simple operation and good performance, and the scheme can achieve small delay while keeping the cost low.

\section{Acknowledgment}

This research is supported by NSF grant ANI-0219110 and CISCO URP.

\section{REFERENCES}

[1] L. Hu and S. S. Rappaport, "Personal Communication Systems Using Multiple Hierarchical Cellular Overlays," IEEE JSAC, vol. 13, no. 2, pp. 406-415, Feb. 1995.

[2] S. Okasaka, S. Onoe, S. Yasuda, and C. Y. Lee, "A New Location Updating Method for Digital Cellular Systems," In Proceedings of IEEE Vehicular Technology Conference (VTC), pp. 345-350, 1991

[3] B. Samadi and W. S. Wong, "Optimization Techniques for Location Area Partitioning," In Proceedings of International Conference Special Seminar of Universal Personal Communications, 1992.

[4] B.-K. Lee and C.-S. Hwang, "A Predictive Paging Scheme Based on the Movement Decision of a Mobile Host," In Proceedings of IEEE Vehicular Technology Conference (VTC), vol. 4, pp. 2158-2162, 1999.

[5] I. F. Akyildiz, J. S. M. Ho, and B. Lin, "Movement-Based Location Update and Selected Paging for PCS Networks," IEEE Transactions on Networking, vol. 4, no. 4, pp. 629-638, Aug. 1996.

[6] J. Tsai and H. Hsiao, "Performance of Movement-Based Location Update and One-Step Paging in Wireless Networks with Sparsely Underlaid Microcells," In Proceedings of IEEE Global Communication Conference, vol. 1, pp. 642-647, 2001.

[7] K. -H. Chiang and N. Shenoy, "Architecture and Schemes for Intelligent Mobility Management in Future Mobile Telecommunication Systems," In Proceedings of IEEE Global Communication Conference, vol. 3, pp. 1463-1467, 2000.

[8] Y.-B. Lin, S. Mohan, and A. Noerpel, "Queueing Priority Channel Assignment Strategies for PCS Hand-Off and Initial Access," IEEE TVT, vol. 43, no. 3, pp. 704-712, Aug. 1994.

[9] D-W Tcha, T-J-Choi, and Y-s Myung, "Location Area Partitioning in a Cellular Radio Network", Journal of the Operational Research Society, vol. 48, pp. 1076-1081, 1997. 